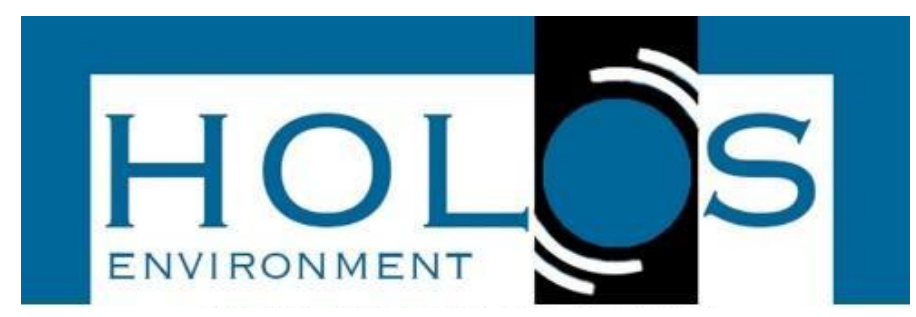

\title{
UMA ABORDAGEM GERAL DOS PRINCIPAIS FATORES INFLUENCIADORES NO DESEMPENHO DE GEOCOMPOSTOS ARGILOSOS
}

\section{A GENERAL APPROACH OF MAIN INFLUENCING FACTORS IN THE GEOSYNTHETIC CLAY LINERS PERFORMANCE}

\author{
Gustavo Dias Miguel ${ }^{1}$; Mozara Benetti ${ }^{1}$; Karla Salvagni Heineck ${ }^{1}$
}

Artigo recebido em: 21/02/2020 e aceito para publicação em: 15/03/2020.

DOI: http://dx.doi.org/10.14295/holos.v20i2.12377

Resumo: Geocompostos argilosos para barreira impermeabilizante (GCL, do inglês: Geosynthetic clay liners) apresentaram-se como uma alternativa a Camadas de Argila Compactada (CCL, do inglês: Compacted Clay Liners). Um produto manufaturado apresenta benefícios em comparação àqueles que se utilizam apenas de produtos naturais. Entretanto, um manejo inadequado destes produtos pode fazer com que estes se tornem inaptos a sua utilização. Se tornando, na verdade, um inconveniente onde aplicado. Assim sendo, algumas considerações básicas devem serem levadas em conta quando de sua utilização. Com isto, aspectos como principais benefícios, resultados de pesquisas, particularidades de sua utilização que prolonguem ou afetem a vida útil e desempenho, são discutidas no presente trabalho. Ao final, é realizada uma breve comparação entre as duas técnicas correlatas, GCLs e CCLs, ambas utilizadas como barreiras impermeabilizantes.

Palavras-chave: Liners. Camada de Proteção. Geosynthetic Clay Liner. GCL. Geossintéticos.

Abstract: Geosynthetic Clay Liners (GCL) are an alternative to Compacted Clay Liners (CCL). Manufactured products present more benefits than those that uses just natural materials. However, a deficient management of GCLs could make this product improperly to use. Becoming indeed, an inconvenient issue where applied. Therefore, some main points should be considered when GCLs were used. Thus, main benefits, important research results, life cycle dependency and performance improvement, were discussed here. Finally, a brief comparison between GCLs and CCLs was carried out.

Keywords: Liners. Protection Layer. Geosynthetic Clay Liners. GCL. Geosynthetics.

\section{INTRODUÇÃO}

A excessiva necessidade de matérias primas e, extrema urgência da utilização destes materiais, contribuíram para o surgimento dos geossintéticos. Estes, como a grande maioria dos produtos que utilizamos hoje em dia, tiveram seu amplo desenvolvimento durante ou logo após à Segunda Guerra Mundial. Em virtude das dificuldades vivenciadas neste período, que demandavam uma resposta rápida e eficaz para as questões que viessem a surgir. Dentre as problemáticas recorrentes deste período citam-se, por exemplo,

1 Universidade do Rio Grande do Sul (UFRGS), Porto Alegre, RS. E-mail: (gustavo.miguel@ufrgs.br, $\underline{\text { mozara.benetti@ufrgs.br, karla.heineck@ufrgs.br) }}$ 
a falta de capacidade de carga de solos sobre os quais, havia-se a necessidade de se transpor como uso de veículos extremamente pesados. Além disto, problemas com a disposição de resíduos pós-guerra, visto que em sua grande maioria, tratava-se de materiais perigosos a saúde humana bem como, outras tantas questões que rodeiam um cenário de guerra. Assim sendo, elementos como as geocélulas, geotêxteis, geocomposto argiloso para barreira impermeabilizante (GCL, do inglês: geosynthetic glay liners) entre outros inúmeros produtos sintéticos, surgiram como alternativa à solução de problemas como estes.

Com o passar dos anos, além da intenção de solucionar problemas como os acima mencionados surge também, um cunho ambiental que acaba impulsionando a utilização de geossintéticos. A extração desenfreada de matérias primas, fez com que jazidas de materiais naturais beirassem a escassez trazendo com isto, danos à fauna e flora destes locais perturbados. A partir daí materiais sintéticos se mostraram como uma alternativa, contribuindo para uma redução e dependência de materiais naturais.

Com isto, com base no até então exposto, este artigo irá focar em discutir aspectos relacionados à Geocomposto Argilosos - GCLs os quais, encaixam-se tanto na resolução da problemática da disposição correta de resíduos como, no auxílio da redução da dependência de materiais naturais. Visto que, a partir do surgimento destes, possibilitou-se uma redução da utilização da metodologia de Camadas de Argilas Compactadas (CCL, do inglês: Compacted Clay Liners). Desta forma, aspectos como principais benefícios, principais resultados encontrados em pesquisas, particularidades de sua utilização que afetem ou prolonguem sua vida útil desempenho serão discutidos aqui. Ao final, uma breve comparação entre as técnicas correlatas, GCLs e CCLs, é fornecida.

\section{GEOCOMPOSTO ARGILOSO IMPERMEABILIZANTE - GCL}

Geocompostos argilosos para barreira impermeabilizante tratam-se de produtos manufaturados em que, onde aplicados, formam uma barreira capaz de conter água, lixiviados e, em alguns casos, gases devido a presença da bentonita ou material similar de baixa condutividade (ESTORNELL; DANIEL, 1992; BOARDMAN; DANIEL, 1996; KOERNER, 2005). Koerner (2005) em concomitância com Shukla e Yin (2006), referem-se a este produto como um composto entre dois ou mais materiais o que justifica sua classificação como um geocomposto. A união entre um ou mais geossintéticos acaba por explorar o que cada um apresenta como vantagem, além disto, um trabalha em benefício 
do outro. Sendo assim, e da mesma forma que Vertematti (2004), as baixas permeabilidades, as boas características de resistência química e mecânica, o controle de fabricação de um produto manufaturado e, a facilidade de instalação, tornam este um produto versátil. Somando-se a isto, Chen et al. (2010) ainda salienta o preço competitivo destes quando comparado a demais métodos, a fina espessura que contribui para sua trabalhabilidade e, um dos pontos mais importantes, a complacência com a ocorrência de recalques diferenciais.

De modo geral, este material tem como principais aplicações problemas relacionados à área de geotecnia ambiental em que, por meio da aplicação deste, buscase mitigar questões que venham a acometer o meio (GIROUD et al. 1997; LAKE; ROWE, 2000; ROWE 2005; BARROSO et al. 2006; FOLTZ et al. 2012; ROWE 2014; ROUF et al. 2016; BOUAZZA et al. 2017). Algumas vantagens e desvantagens deste geossintético são apresentadas na Tabela 1.

Tabela 1 - Vantagens e desvantagens dos GCLs

Vantagens Desvantagens

Rápida instalação/mão de obra não tão Baixa resistência ao cisalhamento da bentonita, quando qualificada/custo baixo. hidratada, para o caso de GCLs não reforçados.

Baixa condutividade hidráulica se corretamente Risco de perfurações geralmente por punção durante ou utilizado. após a instalação.

Boa capacidade de suportar recalques diferenciais.

Possibilidade de perda de parte da bentonita durante a instalação.

Possui capacidade de se autorregenerar.

GCLs com baixa umidade podem permitir a permeabilidade de gases.

Não depende da disponibilidade de solos locais.

Fácil de reparar.

Problemas de resistência de interface com outros materiais.

Pequena capacidade de atenuação de lixiviados.

Resistência ao congelamento e degelo.

Pequena resistência de pós-pico.

Ocupa um menor espaço comparado a técnica de argila compactada, devido sua fina espessura.

Possibilidade de aumento da condutividade hidráulica com o tempo devido a diminuição da espessura da bentonita causado pelas sobrecargas.

Não necessita de teste de condutividade hidráulica.

Possibilidade de aumento da condutividade hidráulica no caso da bentonita não for hidratada corretamente e entrar em contato diretamente com o contaminante.

GCLs hidratados são capazes de atuar como barreiras para gases.

Alto fluxo difuso (migração do local de maior concentração para o local de menor concentração) comparado com CCL.

Auxiliam na melhor distribuição de tensões ao substrato.

Propenso a troca de íons para o caso de GCLs com bentonita sódica, o que poderia vir a interferir na dupla camada difusa do argilomineral.

Propenso a dissecação, causando fissuras e aumentando a permeabilidade.

Fonte: Modificado de Bouazza (2002) 


\subsection{Modelos disponíveis}

Como já mencionado, os GCLs representam compostos providos da união de um ou mais geossintéticos. Onde, neste caso especificamente, evolvem a utilização de geotêxteis ou geomembranas, com a adição de bentonita em uma de suas faces. A bentonita adicionada pode ser do tipo sódica ou cálcica, o que irá depender a que materiais o GCL estará exposto em campo. Dando-se, em geral, preferência para as bentonitas sódicas as quais possuem maior poder de expansão logo, maior poder de impermeabilização.

Os GCLs à base de geotêxteis são confeccionados de forma a produzir um formato de sanduiche, onde a bentonita fica envolta e confinada por duas camadas de geotêxteis. Estes, são divididos em duas grandes áreas, não-reforçados e reforçados. No caso daqueles os quais não possuem reforço, a bentonita é aderida aos geotêxteis por meio de um adesivo. Já os reforçados, sua estrutura é mantida por meio de costura ou então, agulhamento. A opção reforçada tem a intenção de solucionar uma das desvantagens do GCL, a suscetibilidade ao cisalhamento interno. Koerner (2005) e Chen et al. (2010) mencionam que a expansão excessiva do GCL, pode fazer com que a bentonita fique mais suscetível ao cisalhamento interno, desta forma, o reforço atua então neste sentido de aumentar sua resistência.

Outra opção são os GCLs os quais possuem como base geomembranas. Diferente dos anteriormente mencionados, estes não possuem um formato de sanduíche, onde uma única camada de geomembrana é disposta na qual a bentonita é aderida através de adesivos. A Figura 1 exemplifica os modelos de GCLs existentes.

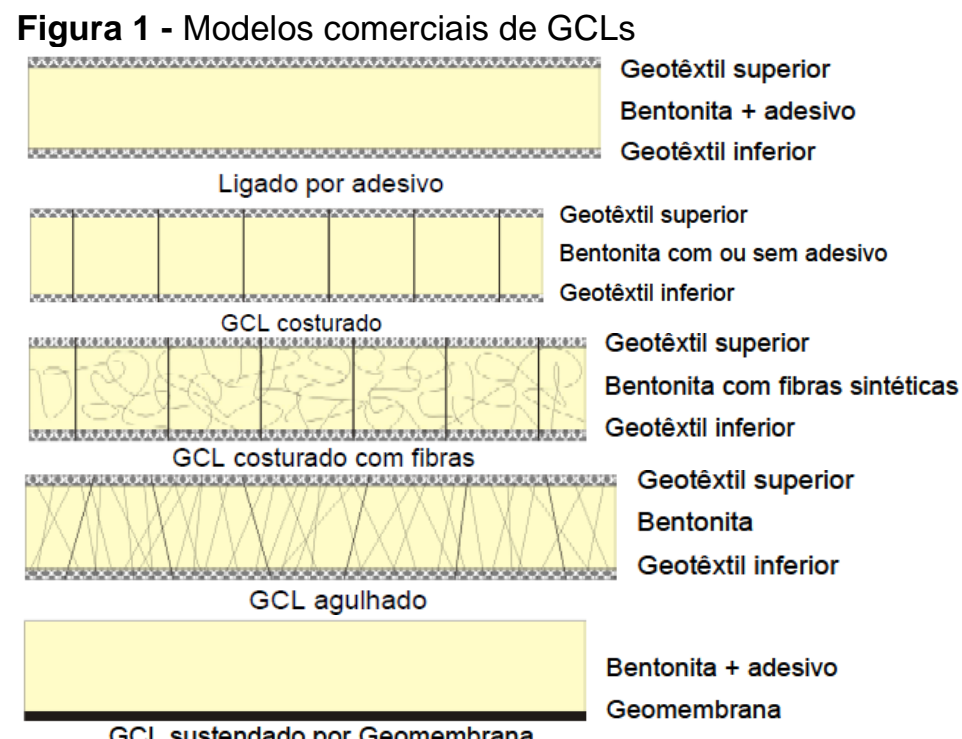

Fonte: Modificado de Mendes (2010) 


\section{PROPRIEDADES GEOTECNICAS DOS LINERS DE BENTONITA}

\subsection{Espalhamento lateral}

Em concomitância com o aumento da fragilidade devido à excessiva expansão da bentonita, surge também a possibilidade da ocorrência do fenômeno conhecido como espalhamento lateral (do inglês: squeezing). O qual, "pode ocorrer se uma carga não perfurante estiver estacionada sobre o GCL com insuficiente solo de cobertura" (KOERNER, 2005). Koernet et al. (1998a, 1998b) afirmam que o espalhamento lateral ou extrusão da bentonita, pode vir a ocorrer quando esta se encontrara úmida e instável. O eventual acontecimento deste fenômeno, acaba por criar um local de alta permeabilidade indo contra ao que se espera quando da aplicação de GCLs (FOX et al, 1997).

Neste sentido, necessita-se garantir uma espessura uniforme deste geossintético como também, uma máxima espessura adequada sem que o mesmo se torne extremamente frágil do ponto de vista de resistência ao cisalhamento. Koerner (2005) apesar de salientar que uma expansão demasiada pode induzir a bentonita ao cisalhamento interno, este também comenta que a espessura do GCL, após sua hidratação, tem papel fundamental acerca da permeabilidade do produto. Ou seja, GCLs com maior ganho de espessura durante sua hidratação, atuarão de forma melhor como barreira impermeabilizante.

Chen et al. (2010) sugere que para minimizar o acontecimento do espalhamento lateral e, por consequência a extrusão da bentonita, produtos como estes sejam aplicados em campo sem uma pré-hidratação, se desenvolvendo ao longo do tempo e a partir da umidade do próprio local. Isto porque, uma sequência construtiva que leva em conta inicialmente uma sobrecarga e, somente após isto, uma hidratação, tende a incorrer em menores perdas de bentonita no interior do produto. Apesar de ser uma alternativa interessante, é na verdade, garantindo-se uma adequada hidratação e expansão que fará com que o produto cumpra com o esperado. Desta forma, a escolha do correto material para camada de cobrimento além de sua correta espessura, serão os fatores que farão com que os problemas acima mencionados não interfiram na eficácia deste produto.

Koerner e Narejo (1995) ao realizarem uma série de ensaios sobre OS GCLs, utilizaram como camada de proteção uma areia bem graduada. Os autores puderam concluir que a espessura aquela que atenda uma razão de cobrimento de $H / B=1,0$ (onde $H$ é a altura do solo de proteção e, $B$ a largura do material perfurante) esta seria a adequada 
as condições de proteção do GCL. Já De Battista e Fox (1996) ao empregarem como camada de cobertura uma areia mal graduada sem pedregulhos ou finos, com diâmetro médio dos grãos de $D_{50}=0,6 \mathrm{~mm}$, estes chegaram à conclusão que na verdade uma razão de $H / B=1,5$ deve ser utilizada. Miguel et al. (2017) variando a forma de aplicação de carga aos GCLs como também, buscando introduzir a possibilidade da utilização de um resíduo oriundo do beneficiamento de ágata $\left(D_{50}=0,42 \mathrm{~mm}\right)$ como camada de proteção, constataram da mesma forma que uma mínima razão de $H / B=1,5$ seja necessária e adequada à proteção de geocompostos argilosos.

\subsection{Resistência à perfuração}

Uma das maiores preocupações relacionadas a GCLs, trata-se da possibilidade de estes virem a desenvolver furos, seja durante o transporte ou como na maioria dos casos, durante sua instalação. As perfurações ocorridas seguem, em geral, o mecanismo de punção, fazendo com que a local onde desenvolvida se tornem pontos de alta permeabilidade. Nos casos da bentonita sódica, devido sua elevada expansão, esta possui a capacidade de auto reparação (ESTORNELL e DANIEL, 1992; BOARDMAN e DANIEL, 1996; DIDIER et al. 2000; ROWE et al. 2018). Didier et al. (2000), por exemplo, mencionam que perfurações de até $30 \mathrm{~mm}$ são capazes de serem seladas após a hidratação do GCL e que, para tamanhos como estes, cerca de 15 dias são necessários. Já Koerner (2005) limita a auto reparação de perfurações a furos que contenham apenas aberturas de até 25 mm. Como a expansão da bentonita está ligada à sua Capacidade de Troca Catiônica (CTC), a presença exagerada de íons adsorvidos em sua superfície pode afetar o processo de auto reparação. Este fato decorre do fato da bentonita possuir elevada CTC e uma grande quantidade de íons em sua superfície irá, por consequência, adsorver uma proporção elevada de cátions trocáveis, podendo ocorrer então na verdade uma floculação destes argilominerais. Consequentemente, uma diminuição em sua capacidade de se auto reparar fato este, reportado por Lin e Benson (2000) e Mazzieri e Pasqualini (2000).

\subsection{Comportamento tensão vs deformação e resistência ao cisalhamento de GCLs}

Em virtude de seus locais de instalação, geocompostos argilosos estão suscetíveis a ação de carregamentos externos. A exemplo disto, podemos mencionar barreiras hidráulicas em fundos de aterros sanitários e recobrimento de taludes, cada uma destas se 
difere pela forma em o carregamento vem a ser aplicado ao produto. No caso da primeira situação, o carregamento se dá, em geral, de forma normal ao plano, por conseguinte, ações compressivas tenderão a assolar o GCL. Já no caso de taludes, por exemplo, os quais possuem uma certa inclinação, surgem também tensões cisalhantes as quais virão a atuar seja na interface geossintético-solo, interface bentonita-geossintético e, ainda, internamente ao GCL, ou seja, diretamente à bentonita. Desta forma, a tensão de cisalhamento a que estes elementos estarão sujeitos é, também, um fator de elevada importância quando do emprego destes materiais.

Tendo-se isto em conta, algumas considerações precisam serem abordadas. Inicialmente, no que diz respeito à interação de um geocomposto argiloso com o solo, isto estará intimamente ligado ao tipo de solo presente na interface, mas também, a forma de instalação e ancoragem adotada (CHIU; FOX, 2004). Solos com partículas mais angulares terão por consequência, uma maior interação entre estes dois materiais logo, uma maior resistência de pico será desenvolvida. Além disto, Garcin et al. (1995), Feki et al. (1997) e Fox e Stark (2004) relatam também, que atingida a resistência de pico entre a interface solo-GCL, esta persistirá em sua magnitude sem quedas significativas, ou seja, a relação entre resistência ao cisalhamento residual e resistência ao cisalhamento de pico se aproxima da unidade $\left(\mathrm{T}_{r} / \mathrm{T}_{\mathrm{p}} \approx 1\right)$, o que ocorre para diferentes tipos de solo e geocompostos argilosos. A eventual queda da resistência de pico para estes casos, será devido a perda de rugosidade desta interface. De mais a mais, apesar da necessidade de se verificar a correta interação entre solo-GCL, esta interface acaba sendo aquela de menor preocupação já que a sustentação deste geossitético passará irrestritamente pelas formas de ancoragem empregadas.

Voltando-se então as demais situações, isto é, interface bentonita-geossintético e resistência ao cisalhamento interno da bentonita, ambas estas estão agora associadas ao modelo de GCL utilizado. Já que a bentonita quando hidratada, tem sua resistência ao cisalhamento reduzida drasticamente o que leva a este material se tornar suscetível a deformações, espalhamento lateral, exsudação, dentre outros problemas associados a esta dada ocorrência.

Nestes casos, poderá haver tanto a manutenção da resistência de pico quanto, a queda drástica desta passando-se então, a prevalecer a resistência residual. Fato este, que pode estar associado ao rearranjo das partículas de argila, perda da rugosidade entre os materiais que compõem o GCL ou até mesmo, o colapso no material como um todo (e.g. arrancamento de uma de suas faces) (FOX; STARK, 2004). 
Assim sendo, quando utilizados geocompostos argilosos reforçados, isto é, agulhados ou por meio da inserção de fibras, os quais intencionam suprimir a fragilidade da bentonita devido sua hidratação, nestas situações a falha interna devido a tensões cisalhantes poderá vir a se desenvolver nestes reforços. Desta forma, a resistência de pico estaria associada a resistência dos reforços enquanto a resistência residual, após ruptura dos reforços, a resistência remanescente do conjunto como um todo (FOX, STARK, 2004; SIMON; MÜLLER, 2004; MÜLLER et al. 2008; VUKELIĆ et al. 2008). Agora, se tratando de GCLs costurados ou colados por meio de adesivos, as falhas internas costumam a ocorrer próximo às costuras, ou então, devido ao arrancamento de um dos geotêxteis onde fora empregado o adesivo (FOX; STARK, 2004; FOX; STARK, 2015).

Se inicialmente, nos determos a compreensão de geocompostos argilosos nãoreforçados, no que diz respeito ao seu comportamento tensão vs deformação, para Stark et al. (1998) e Fox e Stark (2004) estes modelos de GCL se mostram inapropriados a sua utilização em taludes ou locais onde se tenha uma tensão cisalhante transversalmente aplicada aos mesmos. Isto porquê, estes produtos, sem reforços, além de possuírem uma baixa resistência ao cisalhamento, da mesma forma não apresentam uma resistência residual significativa. Locais de elevados recalques, como aterros sanitários onde há decomposição contínua dos materiais, faz-se pertinente a aplicação de GCLs que possuam uma elevada resistência residual.

Se tratando agora de geocompostos argilosos reforçados, o agulhamento, a inserção de fibras ou costura acaba por gerar um confinamento extra a estes materiais (Fox e Stark, 2004). Devido ao potencial expansivo da bentonita, está ao tentar expandir será reprimida em virtude dos reforços, o que de certa forma, acaba sendo convertido em um aumento da tensão efetiva média ( $p)$. Um aumento da tensão efetiva resultará, por consequência, em um aumento da resistência destes compostos. Além disto, a presença destas fibras faz também, com que se tenha uma menor retenção de água por parte da bentonita logo, tornando está menos suscetível ao cisalhamento e ao espalhamento lateral (FOX; STARK, 2004). Ao compararmos diferentes modelos GCLs reforçados, como por exemplo, exemplares agulhados e com inclusão de fibras com aqueles, que possuem apenas costura e adesivo, estes últimos mesmo reforçados, podem vir a ter uma resistência ao cisalhamento de pico cerca de $50 \%$ menor do que os primeiros. Isto claro, sobre condições de tensões normais idênticas para ambos os modelos (FOX; STARK, 2004).

Já dentre as diferenças entre GCLs reforçados e não-reforçados, além é claro de uma maior resistência por parte dos primeiros, os mesmos acabam também por possuir 
uma baixa relação $T_{r} / T_{p}$, ou seja, após atingido o pico de resistência há uma queda brusca desta, com a resistência ao cisalhamento residual diferindo significativamente daquela obtida no pico. Isto, devido a presença dos reforços, onde a queda de resistência está associada à sua ruptura. Desta forma, pode-se dizer que GCLs que utilizam adesivo são mais dúcteis e, com isto, permitem uma maior deformação previamente à tensão máxima (FOX; STARK, 2004).

Por fim, nos detendo a resistência ao cisalhamento destes compostos e, a resistência ao cisalhamento propriamente da bentonita, há uma dependência destes a magnitude da tensão normal efetiva atuante, similar ao que ocorre nos solos em geral. Sendo, portanto, tanto maior a resistência ao cisalhamento de pico e residual quanto maior a tensão normal efetiva aplicada (FOX; STARK, 2004). Da mesma forma, observa-se uma dependência do ângulo de atrito interno as magnitudes de tensão normal efetiva aplicadas, relação esta que se dá de maneira inversamente proporcional, ou seja, quanto maior a tensão normal efetiva menor o ângulo de atrito interno (FOX; STARK, 2004). Com base nestas constatações, a prática de engenharia atualmente vem caracterizando parâmetros de resistência de GCLs em termos de tensões normais totais, independentemente de estes terem sido já considerados elementos que permitam a drenagem (GILBERT et al., 1997). Parâmetros estes, adotados em análises de estabilidades em tensões efetivas, por mais conservativo que possa parecer.

\subsection{Condutividade hidráulica}

Como já mencionado, a condutividade hidráulica do GCL está associada a expansão da bentonita, salvo casos aqueles os quais utilizam como base uma geomembrana. Em geral, a condutividade hidráulica de GCLs varia da ordem de $10^{-10}$ a $10^{-12} \mathrm{~m} / \mathrm{s}$ (Tabela 2 2) muito abaixo do exigido por muitos normativos, que exigem na maioria das vezes permeabilidades da ordem de $10^{-7} \mathrm{~m} / \mathrm{s}$ para barreiras impermeabilizantes de aterros sanitários (liners de fundo). Esta variação de permeabilidade pode estar associada a qualidade dos geotêxteis utilizados, da bentonita, bem como, vir a depender da tensão de confinamento aplicada ao GCL durante sua hidratação. Bouazza (2002) reuniu uma série de resultados que envolviam GCLs hidratados a diferentes tensões de confinamento (Figura 2), com a unificação destes resultados, pode-se perceber uma grande influência por parte da tensão de confinamento. Onde GCLs submetidos a tensões mais elevadas durante sua hidratação, resultaram em uma menor condutividade hidráulica. Petrov et al. (1997) 
acreditam que isto esteja relacionado a diminuição do índice de vazios, visto que a condutividade hidráulica das barreiras de GCL é governada em grande parte pelo índice de vazios da bentonita após a hidratação. Koerner (2005) comenta também, que GCLs sem qualquer tensão de confinamento no decorrer de sua hidratação, terão após esta, uma menor resistência ao cisalhamento interno, salientando com isto a importância deste procedimento.

Tabela 2 - Variação da condutividade hidráulica de

\begin{tabular}{|c|c|}
\hline $\begin{array}{c}\text { Permeabilidade } \\
(\mathrm{cm} / \mathrm{s})\end{array}$ & Trabalho \\
\hline $3 \times 10^{-11}$ & Miguel et al. (2017) \\
\hline $3,8 \times 10^{-11}$ & Rowe et al. (2017) \\
\hline $4,4 \times 10^{-11}$ & Rowe et al. (2017) \\
\hline $4,3 \times 10^{-11}$ & Rowe et al. (2017) \\
\hline $5,9 \times 10^{-11}$ & Rowe et al. (2017) \\
\hline $6-7 \times 10^{-9}$ & Melchior et al. (2010) \\
\hline $6-8 \times 10^{-11}$ & Benson et al. (2007)a \\
\hline $2 \times 10^{-7}$ e $8 \times 10^{-8}$ & Benson et al. (2007) ${ }^{\mathrm{b}}$ \\
\hline $1-9 \times 10^{-7}$ & Benson et al. $(2007)^{c}$ \\
\hline $10^{-8}$ a $10^{-10}$ & Estornell e Daniel (1992) \\
\hline $10^{-9}$ & Petrov e Rowe (1997) ${ }^{d}$ \\
\hline $10^{-6}$ & Petrov e Rowe (1997) \\
\hline
\end{tabular}

a permeabilidade original

${ }^{\mathrm{b}}$ permeabilidade após 2 anos

c permeabilidade após 5 anos

d permeabilidade quando hidratado com água

e permeabilidade quando hidratado com 2 Molar de $\mathrm{NaCl}$

Figura 2 - Relação entre a condutividade hidráulica de GCLs e a tensão confinante aplicada

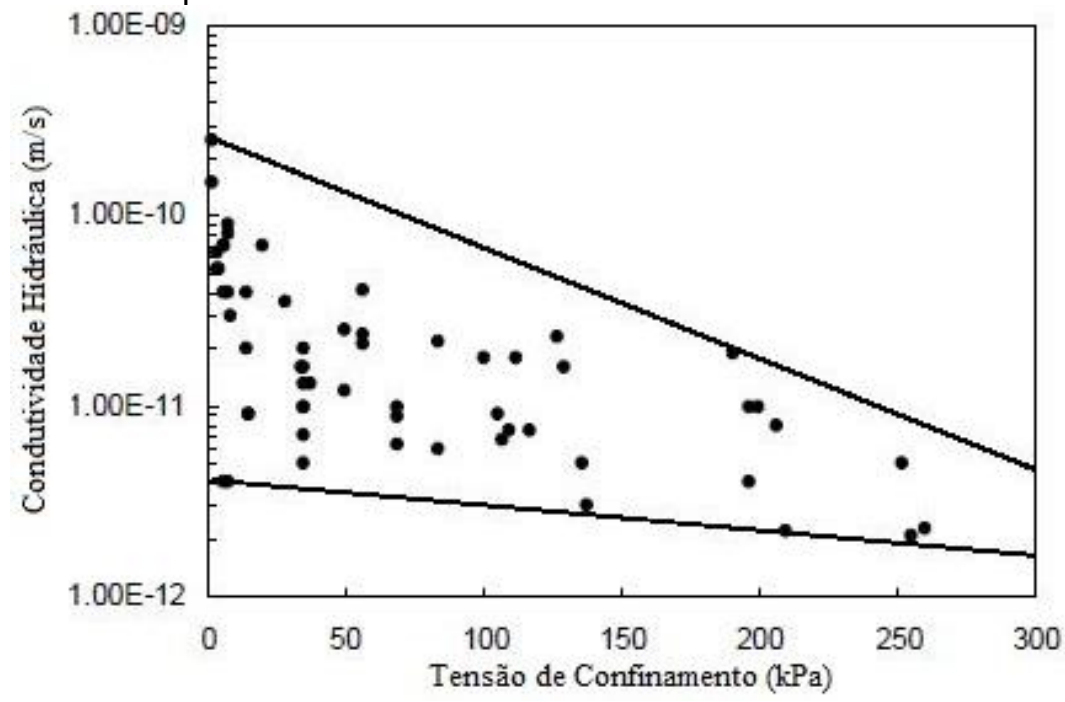

Fonte: Modificado de Bouazza (2002) 
Shackelford et al. (2000) discutiram os fatores que afetam a condutividade hidráulica de GCLs permeados com líquidos diferentes do padrão (água). Os resultados do ensaio mostraram que líquidos não convencionais que contêm altas concentrações de cátions monovalentes e baixas concentrações de cátions bivalentes podem causar aumentos significativos na condutividade hidráulica desde que o ensaio seja realizado suficientemente longo para permitir a troca de cátions adsorvidos. Kolstad et al (2004) comparou a expansão da bentonita sódica e sua condutividade hidráulica em ensaios com GCL convencional (sem pré hidratação) e o GCL pré hidratado pelo fabricante, com teor de água de $43 \%$ na amostra. Os ensaios utilizaram soluções inorgânicas agressivas para a hidratação dos GCLs. Os autores perceberam que a grande vantagem do GCL pré hidratado, tanto na questão do inchamento, quanto na condutividade hidráulica, tendo seus valores próximos a quando a hidratação é feita com água destilada.

Lee et al. (2005) avaliaram a influência das correlações a partir das alterações nas propriedades físicas e o aumento da condutividade hidráulica, a qual acaba sendo uma função da propriedade física, da qualidade da bentonita e da tensão efetiva usada na medição da condutividade hidráulica. O autor observou que dentre os índices físicos, o limite de liquidez foi considerado o mais sensível entre os índices analisados (limite de liquidez, volume de sedimentação e índice de dilatação) em que maiores reduções no índice de dilatação e no volume de sedimentação em relação ao limite de liquidez foram necessários para que aumentos substanciais de condutividade hidráulica ocorressem. Os resultados deste estudo também mostraram que a tensão efetiva usada nos ensaios de condutividade hidráulica desempenha um papel significativo em termos da correlação entre uma propriedade física e a condutividade hidráulica.

Meer e Benson (2007) explicam que a bentonita é sensível às interações químicas com o liquido hidratante e a troca de íons que ocorre na bentonita pode alterar significativamente suas propriedades físicas. Estudos sobre GCLs extraídos de fundos de aterros mostraram que a baixa condutividade hidráulica da bentonita sódica não é necessariamente mantida durante a vida útil do projeto. Os autores encontraram valores de condutividade hidráulica na faixa de $10^{-7}$ a $10^{-6} \mathrm{~m} / \mathrm{s}$ para GCLs desenterrados após certo tempo de serviço. As altas condutividades hidráulicas observadas nestes GCLs foram atribuídas à perda de capacidade de expansão da bentonita juntamente com a formação de rachaduras e outras deformações macroscópicas que ocorrem durante a desidratação e ressecamento. 
Zanella (2019) avaliou a eficiência impermeabilizante, ao longo do tempo, de uma barreira constituída por um geocomposto bentonítico (GCL) ao ser permeado com biodisel B100 e observou que a condutividade hidráulica teve aumento de permeabilidade da escala $10^{-11} \mathrm{~m} / \mathrm{s}$ até a escala $10^{-9} \mathrm{~m} / \mathrm{s}$. Observando um decréscimo na capacidade de impermeabiliação do material ao longo do tempo. Di Emidio et al. (2011), explica que a exposição a soluções orgânicas ou inorgânicas altamente concentradas, pode alterar quimicamente a argila aumentando a condutividade hidráulica. Quando GCLs são permeados com água, a condutividade hidráulica tende a ser menor (ou seja, tipicamente $\mathrm{k}<3 \times 10^{-\mathrm{m}} \mathrm{cm} / \mathrm{s}$ ) e isso se dá devido à bentonita, que incha na presença de água, resultando em constrição dos caminhos de fluxo entre as partículas de argila ou agregados de partículas (DANIEL et al., 1997).

\section{HIDRATAÇÃO E EROSÃO INTERNA}

O líquido de hidratação tem influência preponderante no posterior desempenho do GCL, já que é sua correta hidratação que irá ditar sua espessura final bem como, sua capacidade de atuar como barreira hidráulica. Desta forma, estes produtos precisam ter sua hidratação garantida para que exerça sua função. Koerner (2005) indica que para obtenção da máxima expansão da bentonita, água destilada é recomendada, ou então, em substituição desta, água de abastecimento público. Deve-se ainda, se tomar o cuidado quando da instalação do GCL, que este só venha a ter contato com derivados de petróleo e chorume após sua completa hidratação por água. A hidratação de GCLs com outros produtos, diferente dos recomendados, culmina em uma não expansão da bentonita logo, em altas permeabilidades (KOERNER, 2005).

Bouazza (2002) menciona que as principais características do líquido permeante, que influenciam na condutividade hidráulica de GCLs, são a existência concentrada de cátions monovalentes e polivalentes. Quando estes forem monovalentes, há a presença da fase cristalina e hidratação osmótica, resultando em um maior distanciamento entre os argilominerais. Com isto, uma maior expansão é observada. Do contrário, quando polivalentes, deixa-se de existir a hidratação osmótica devido à alta atração eletroestática entre os argilominerais, podendo haver redução e até floculação destes últimos (HEINECK, 2013).

Se tratando do período de hidratação, Miguel et al. (2017) adotaram um tempo mínimo de 24 horas, mesmo tempo utilizado nos estudos de Koerner e Narejo (1995) e De 
Battista e Fox (1996). No entanto, em paralelo a determinação da camada de cobertura adequada para GCLs, Miguel et al. (2017) também realizaram ensaios de hidratação de geocompostos argilosos e assim, buscar determinar a influência do tempo de exposição destes ao líquido de hidratação. A partir disto, percebeu-se que períodos acima de 24 horas - até 72 horas - pouco influem na expansão da bentonita sendo assim, desnecessário a adoção de períodos superiores ao estabelecido como mínimo. Para o caso destes autores, após 24 h de hidratação por água de abastecimento público, o GCL utilizado apresentou uma expansão média de cerca de 1,6 vezes o seu tamanho original.

Um GCL quando manufaturado, sai de fábrica contendo uma determinada quantidade de bentonita por unidade de área (e.g. $4100 \mathrm{~g} / \mathrm{m}^{2}$, MIGUEL et al., 2017). No decorrer de seu transporte e instalação, parte desta massa acaba se perdendo devido granulometria extremamente fina do material. Entretanto, existem também outras demais possibilidades da perda de massa por parte do GCL como por exemplo, nos casos em que estes são instalados sobre subleitos pedregulhosos. Nos quais, na presença de um alto gradiente hidráulico, poderá haver carreamentos de partículas de bentonita através dos geotêxteis. Situações como estas, podem ocorrer por exemplo, nos casos em que há instalação de GCLs próximos a drenos, ou então, quando utilizados como detectores de vazamentos. Em ambos os casos, os quais podem se tratar de líquidos inofensivos ou até mesmo chorume, visando seu posterior tratamento, nestas situações devido à diferença de carga hidráulica de um meio para o outro, poderão haver carreamento das partículas. $O$ que acaba resultando em duas grandes problemáticas, primeiro pelo desarranjo do GCL que perde sua função e, segundo, pela possibilidade de colmatação do dreno que, por consequência, da mesma forma deixa de exercer sua função. Estornell e Daniel (1992) sugerem a utilização de uma ou mais camadas de geotêxteis sobrepostas ao GCL, a fim de atuarem como camadas protetoras deste geossintético impermeabilizante e impedirem a perda de massa por parte destes.

\section{GEOCOMPOSTO ARGILOSO IMPERMEABILIZANTE (GCL) VERSUS CAMADA DE ARGILA COMPACTADA (CCL)}

Durante anos a principal técnica empregada como barreira impermeabilizante baseava-se exclusivamente na confecção de uma Camada de Argila Compactada (CCL, do inglês: Compacted Clay Liners). Esta consiste na escolha de um material natural com características de baixa permeabilidade onde a estes, são empregados tratamentos como 
peneiramento, destorroamento, dentre outros, até virem a ser dispostos no local e então compactados. Com o surgimento dos GCLs, despontou ao mesmo tempo, o questionamento de qual das metodologias seria a mais eficaz ou então, teria um melhor desempenho frente as possíveis aplicações. Rowe (1998, 2001) e Bouazza (2002), consideram que a comparação entre estas duas técnicas é algo extremamente relativo e de difícil realização. Visto que, um grande número de considerações e características do local deve ser levado em conta, como por exemplo: a quantidade e concentração de poluente existente, o qual poderá possivelmente vir a atacar a barreira, nível do lençol freático, dentre outras tantas peculiaridades que estão associadas aos locais de utilização. Em posse destas informações, emergem daí questionamentos como, considerações quanto a superfície específica dos grãos de argilas, suas cargas e agrupamentos químicos que irão quantificar sua reatividade, a mensuração de sua CTC que irá nortear a capacidade de expansão do argilomineral, dentre outros. Com isto, percebe-se que a simples comparação entre uma técnica e outra não é algo fácil mais ainda, não deve permanecer em algo tão somente superficial, devendo-se desta forma, englobar os mais diversos questionamentos possíveis. Bouazza (2002) arrisca-se a dizer que na maioria dos casos, a performance de GCLs é equivalente à CCLs ainda, que em alguns critérios, chega até superar o desempenho deste último. Entretanto, levando-se em consideração problemas relacionados ao fluxo de contaminantes logo, a compatibilidade química destes com a barreira, CCLs permanecem ainda demonstrando maior viabilidade. Manassero et al. (2000) busca realizar esta comparação de forma qualitativa, a qual é exposta através da Tabela 3.

Tabela 3 - Comparação qualitativa entre GCLs e CCLs

\begin{tabular}{|c|c|c|c|c|c|}
\hline \multirow{2}{*}{ Categoria } & \multirow[t]{2}{*}{ Critério de avaliação } & \multicolumn{3}{|c|}{ Equivalência do GCL para o CCL } & \multirow[b]{2}{*}{$\begin{array}{l}\text { Dependente } \\
\text { do local de } \\
\text { instalação } \\
\text { ou do } \\
\text { produto } \\
\text { utilizado }\end{array}$} \\
\hline & & $\begin{array}{l}\text { GCL } \\
\text { provavelmente } \\
\text { superior }\end{array}$ & $\begin{array}{l}\text { GCL } \\
\text { provavelmente } \\
\text { equivalente }\end{array}$ & $\begin{array}{l}\text { GCL } \\
\text { provavelmente } \\
\text { inferior }\end{array}$ & \\
\hline \multirow{5}{*}{$\begin{array}{l}\text { Problemas } \\
\text { construtivos }\end{array}$} & Facilidade de instalação & $\mathrm{X}$ & & \multirow{5}{*}{$\mathrm{X}$} & \\
\hline & $\begin{array}{l}\text { Disponibilidade de } \\
\text { material }\end{array}$ & $\mathrm{X}$ & & & \\
\hline & $\begin{array}{l}\text { Resistência a punção } \\
\text { Qualidade assegurada }\end{array}$ & $\mathrm{X}$ & & & \\
\hline & $\begin{array}{ll}\text { Velocidade } & \text { de } \\
\text { construção } & \end{array}$ & $x$ & & & $\mathrm{X}$ \\
\hline & $\begin{array}{l}\text { Condições do substrato } \\
\text { Necessidade de água }\end{array}$ & $\mathrm{x}$ & & & $\mathrm{X}$ \\
\hline
\end{tabular}


Tabela 3 - Comparação qualitativa entre GCLs e CCLs

(conclusão)

\begin{tabular}{|c|c|c|c|c|c|}
\hline \multirow[t]{2}{*}{ Categoria } & \multirow[t]{2}{*}{ Critério de avaliação } & \multicolumn{3}{|c|}{ Equivalência do GCL para o CCL } & \multirow[b]{2}{*}{$\begin{array}{l}\text { Dependente } \\
\text { do local de } \\
\text { instalação } \\
\text { ou do } \\
\text { produto } \\
\text { utilizado }\end{array}$} \\
\hline & & $\begin{array}{l}\text { GCL } \\
\text { provavelmente } \\
\text { superior }\end{array}$ & $\begin{array}{l}\text { GCL } \\
\text { provavelmente } \\
\text { equivalente }\end{array}$ & $\begin{array}{l}\text { GCL } \\
\text { provavelmente } \\
\text { inferior }\end{array}$ & \\
\hline & Restrições de clima & & & & \\
\hline \multirow[t]{2}{*}{$\begin{array}{ll}\text { Problemas } & d e \\
\text { transporte } & d e \\
\text { contaminantes } & \end{array}$} & $\begin{array}{l}\text { Capacidade } \\
\text { atenuação }\end{array}$ & & & $X^{a}$ & $x$ \\
\hline & $\begin{array}{l}\text { Permeabilidade a gases } \\
\text { Fluxo do soluto e tempo } \\
\text { de transposição }\end{array}$ & $X^{b}$ & & $x$ & $x$ \\
\hline \multirow{2}{*}{$\begin{array}{l}\text { Problemas } \\
\text { hidráulicos }\end{array}$} & Compatibilidade & $X^{b}$ & & $X$ & \\
\hline & $\begin{array}{l}\text { Água para consolidação } \\
\text { Fluxo constante de água } \\
\text { Tempo de transposição } \\
\text { da água }\end{array}$ & $x$ & $X$ & & \\
\hline \multirow[t]{2}{*}{$\begin{array}{l}\text { Problemas } \\
\text { físicos/mecânicos }\end{array}$} & Capacidade de suporte & & & & $x$ \\
\hline & $\begin{array}{l}\text { Erosão } \\
\text { Gelo-degelo } \\
\text { Recalque total } \\
\text { Recalque diferencial } \\
\text { Estabilidade de taludes } \\
\text { Molhagem-secagem }\end{array}$ & $\begin{array}{l}x \\
x \\
x\end{array}$ & $x$ & & $x$ \\
\hline
\end{tabular}

Fonte: Modificado de Manassero et al. (2000)

\section{CONCLUSÕES}

A partir do surgimento de GCLs, muito se evolui nas questões que envolvem confecções de barreiras hidráulicas no mundo todo em particular no Brasil. Este geossintético tornou-se ainda mais popular após a implementação de políticas como a Política Nacional de Resíduos Sólidos, a qual orienta a disposição ambientalmente adequada para resíduos finais que não comtempla a reciclagem. Porém, como já mencionado, como qualquer outro produto os GCLs possuem suas vantagens, mas também, suas desvantagens. Esta podem vir a serem minimizadas quando se adotado padrões pré-estabelecidos que tem por base, normativos técnicos, ou então, a literatura científica mundial. Neste artigo, algumas das principais considerações com relação a GCLs foram destacadas, tomando-se como base os principais periódicos da área.

Destacou-se aqui, por exemplo, a importância da manutenção da estrutura do GCL pois, este quando sujeito a uma redução da quantidade de bentonita em seu interior, acaba 
por aumentar o fluxo hidráulico. Fato este, que pode também ocorrer devido ao fenômeno de espalhamento lateral da bentonita após sua hidratação. Desta forma, tendo como base estes problemas, bem aqueles relacionados à possibilidade de perfurações, recomenda-se a utilização de uma camada protetora para este geossintético. O que pode ser feito por meio de um solo de granulometria regular e, isento de pedregulhos, com uma razão de cobrimento mínima de $H / B=1,5$. Também, um cuidado especial durante o transporte e instalação destes produtos irá garantir sua completa eficiência.

Quanto a condutividade hidráulica, ficou claro a importância de se hidratar o GCL sob uma tensão confinante (quanto maior a tensão confinante menor sua permeabilidade) procedimento este, um tanto complicado de se obter em campo. Uma alternativa a este impasse, diz respeito a utilização de colunas d'água sobre estes elementos, as quais irão produzir um efeito de sobrepeso sobre o GCL. Quando da utilização de sobrepeso, há necessidade de se garantir um substrato isento de materiais perfurantes. Já quando se tratando da forma de hidratação dos GCLs, recomenda-se a utilização de água destilada, porém, devido à dificuldade muitas vezes de obtenção desta, abre-se a possibilidade para utilização de água de abastecimento público. É importante, previamente a utilização da água de abastecimento público, garantir que esta seja isenta de quaisquer eventuais produtos inibidores da expansão da bentonita. Além disto, recomenda-se uma préhidratação do GCL previamente ao contato como lixiviados e contaminantes. A hidratação realizada por líquidos como estes, pode incorrer em uma contração da dupla camada difusa aumentando assim, a permeabilidade do GCL.

Com relação a erosão interna do GCL, previamente sua instalação, é de suma importância estar a par da possibilidade de existência de fluxos d'água bem como, dos gradientes por estes exercidos. Também, indica-se a não instalação de GCLs sobre materiais muito drenantes como cascalhos e areias, suscetíveis a percolação de água. Além disto, é sugerida a utilização de geotêxteis sobrepostos aos GCLs, visando dificultar o carreamento das partículas.

Por fim, a comparação entre GCLs e CCLs acaba sendo algo de difícil realização e, muitas vezes sendo realizada de maneira abstrata, sem levar em conta as condições do local em que as técnicas serão utilizadas. Em ambos os casos emergem vantagens e desvantagens, no entanto, o que se tem observado atualmente é a utilização em conjunta de ambas as técnicas. Isto, com o intuito de se obter uma barreira de melhor qualidade, onde o benefício da presença do GCL passa por uma redução significativa da camada de CCL podendo com isto, reduzirem-se custos e extração de matérias primas naturais. 


\section{REFERÊNCIAS}

BARROSO, M.; TOUZE-FOLTZ, N.; VONMAUBEUGE, K.; PIERSON, P. Laboratory investigation of flow rate through composite liners consisting of a geomembrane, a GCL and a soil liner.

Geotextiles and Geomembranes, v. 24, n. 3, p. 139-155,

2006. https://doi.org/10.1016/i.geotexmem.2006.01.003

BENSON, C.; THORSTAD, P.; JO, H.; ROCK, S. Hydraulic performance of geosynthetic clay liners in a landfill final cover. Journal of Geotechnical and Geoenvironmental Engineering, v. 133, n. 7, p. 814-827, 2007. https://doi.org/10.1061/(ASCE)1090-0241(2007)133:7(814).

BOARDMAN, B. T.; DANIEL, D. E. Hydraulic Conductivity of Desiccated Geosynthetic Clay Liners. Journal of Geotechnical Engineering, V. 122, n. 3, p. 204-208,

1996. https://doi.org/10.1061/(asce)0733-9410(1996)122:3(204)

BOUAZZA, A. Geosynthetic Clay Liners. Geotextiles and Geomembranes, v. 20, n. 1, p. 3-17, 2002. https://doi.org/10.1016/S0266-1144(01)00025-5

BOUAZZA, A.; ALI, M. A.; ROWE, R. K.; GATES, W. P.; EL-ZEIN, A. Heat mitigation in geosynthetic composite liners exposed to elevated temperatures. Geotextiles and Geomembranes, v. 45, n. 5, p. 406-417, 2017. https://doi.org/10.1016/i.geotexmem.2017.05.004

CHEN, Y-m.; LIN, W-a.; ZHAN, T. L. T. Investigation of mechanisms of bentonite extrusion from GCL and related effects on the shear strength of GCL/GM interfaces. Geotextiles and

Geomembranes, v. 28, n. 1, p. 63-71, 2010. https://doi.org/10.1016/j.geotexmem.2009.09.006

CHIU, P.; FOX, P. J. Internal and interface shear strengths of unreinforced and needle-punched geosynthetic clay liners. Geosynthetics International, v. 11, n. 3, p. 176-199, 2004. https://doi.org/10.1680/gein.2004.11.3.176

DE BATTISTA, D. J.; FOX, P. J. Discussion to "Bearing capacity of hydrated geosynthetic clay liners" by KORNER, R. M.; NAREJO, D. (1995), Journal of Geotechnical Engineering, v. 122, n. 12, p. 1023-1024, 1996. https://doi.org/10.1061/(ASCE)0733-9410(1996)122:12(1023.3).

DANIEL, D.; BOWDERS, J.; GILBERT, R. Laboratory Hydraulic Conductivity Testing of GCLs in Flexible-Wall Permeameters. Testing and Acceptance Criteria for Geosynthetic Clay Liners, edited by WELL, L. (West Conshohocken, PA: ASTM International). 1997. p. 208229. https://doi.org/10.1520/STP11804S

DIDIER, G.; AL NASSAR, M.; PLAGNE, V.; CAZAUX, D. Evaluation of Self-Healing Ability of Geosynthetic Clay Liners. In: ISRM INTERNATIONAL SYMPOSIUM FOR ROCK MECHANICS AND ROCK ENGINEERING, Melbourne, Australia. [Proceedings...]. CRC Press, 2000. p. 1-7.

DI EMIDIO, G.; VAN IMPE, W. F.; VERÁSTEGUI FLORES, R. D. F. Advances in Geosynthetic Clay Liners: Polymer Enhanced Clays. In: Geo- Frontiers Congress, Dallas, Texas, USA. [Proceedings...] . ASCE, 2011. p. 1931-.1940. https://doi.org/10.1061/41165(397)197

ESTORNELL, P.; DANIEL, D. E. Hydraulic Conductivity of Three Geosynthetic Clay Liners. Journal of Geotechnical Engineering, v. 118, n. 10, p. 1592-1606, 1992.

https://doi.org/10.1061/(ASCE)0733-9410(1992)118:10(1592)

FEKI, N.; GARCIN, P.; FAURE, Y. H.; GOURC, J. P.; BERROIR, G. Shear strength of geosynthetic clay liner systems. In: GEOSYNTHETICS ‘97, IFAI, Long Beach, CA.

[Proceedings...]. v. 2, p. 899-912, 1997. 
FOLTZ, N. T-.; ZANZINGER, H.; KOERNER, R. M. Special issue on GCL. Geotextiles and Geomembranes, v. 33, p. P1, 2012. https://doi.org/10.1016/j.geotexmem.2012.02.001

FOX, P.; DE BATTISTA, D. J; CHEN, S-H. A study of the CBR Bearing Capacity Test for Hydrated Geosynthetic Clay Liners. Part of: Testing and Acceptance Criteria for Geosynthetic Clay Liners, edited by WELL, L. (West Conshohocken, PA: ASTM International). 1997. p. 251264. https://doi.org/10.1520/STP11807S

FOX, P. J.; ROWLAND, M. G.; SCHEITHE, J. R. Internal shear strength of three geosynthetic clay liners. Journal of Geotechnical and Geoenvironmental Engineering, v. 124, n. 10, p. 933-944, 1998. https://doi.org/10.1061/(ASCE)1090-0241(1998)124:10(933)

FOX, P. J.; STARK, T. D. State-of-the-art report: GCL shear strength and its measurement. Geosynthetics International, v. 11, n. 3, p. 141-175,

2004. https://doi.org/10.1680/gein.2004.11.3.141

FOX, P. J.; STARK, T. D. State-of-the-art report: GCL shear strength and its measurement - tenyear update. Geosynthetics International, v. 22, n. 1, p. 3-47,

2015. https://doi.org/10.1680/gein.14.00030

GARCIN, P.; FAURE, Y. H.; GOURC, J. P.; PURWANTO, E. Behavior of geosynthetic clay liner (GCL): laboratory tests. In: INTERNATIONAL SYMPOSIUM ON LANDFILLS, $5^{\text {th }}$., 1995. Cagliari. Proceedings [...]. v. 1, p. 347-358, 1995.

GILBERT, R. B.; FERNANDEZ, F.; HORSFIELD, D. W. Shear strength of reinforced geosynthetic clay liner. Journal of geotechnical engineering, v. 122, n. 4, p. 259-266, 1996.

https://doi.org/10.1061/(asce)0733-9410(1996)122:4(259)

GILBERT, R. B.; SCRANTON, H. B.; DANIEL, D. E. Shear strength testing for geosynthetic clay liners. Testing and Acceptance Criteria for Geosynthetic Clay Liners, STP 1308, Well, L. W., Editor, ASTM International, West Conshohocken, PA, pp. 121-135.

GILBERT, R. B.; SCRANTON, H. B.; DANIEL, D. E. Shear Strength Testing for Geosynthetic Clay Liners. Part of: Testing and Acceptance Criteria for Geosynthetic Clay Liners, edited by WELL L. (West Conshohocken, PA: ASTM International). 1997. p. 121-

135. https://doi.org/10.1520/STP11798S

GIROUD, J. P.; BADU-TWENEBOAH, K.; SODERMAN, K. L. Comparison of Leachate Flow Through Compacted Clay Liners in Landfill Liner Systems. Geosynthetics International, v. 4, n. 3-4, p. 391-431, 1997. https://doi.org/10.1680/gein.4.0100

HEINECK, K. S. Material didático desenvolvido para a disciplina de geotecnologia ambiental: geotecnologia ambiental. Porto Alegre: Universidade Federal do Rio Grande do Sul (UFRGS), p. 84, 2013.

KOERNER, R. M. Designing with Geosynthetics. 3. ed. Prentice Hall, Upper Saddle River, New Jersey, USA, 1994. 783 p.

KOERNER, R. M. Designing with Geosynthetics, 5. ed. Pearson, New Jersey, USA, 2005. 796 p.

KOERNER, R. M.; NAREJO, D. Bearing capacity of hydrated geosynthetic clay liners. Journal of Geotechnical Engineering, v. 121, n. 1, p. 82-85, 1995. https://doi.org/10.1061/(ASCE)07339410(1995)121:1(82) 
KOERNER, R. M.; CARSON, D. A.; DANIEL, D. E.; BONAPARTE, R. Update of the Cincinnati GCL test plots. In: Geo-Bento, France. [Proceedings ...]. Insatec Publications, 1998a, p. 291-315.

KOERNER, R. M.; SOONG, T-Y.; GONTAR, A. Selected aspects of GCL shear strength testing. In: Geo-Bento, France. [Proceedings...]. Insatec Publications, 1998b, p. 97-110.

KOLSTAD, D. C.; BENSON, C. H.; EDIL, T. B. Hydraulic conductivity and swell of nonprehydrated geosynthetic clay liners permeated with multispecies inorganic solutions. Journal of Geotechnical and Geoenvironmental Engineering, v. 130, n. 12, p. 1236-1249, 2004.

https://doi.org/10.1061/(ASCE)1090-0241(2004)130:12(1236)

LAKE, C. B.; ROWE, R. K. Swelling characteristics of needlepunched, thermally treated geosynthetic clay liners. Geotextiles and Geomembranes, v. 18, n. 2-4, p. 77-101, 2000. https://doi.org/10.1016/s0266-1144(99)00022-9

LEE, J-M.; SHACKELFORD, C. D.; BENSON, C. H.; JO, H-Y.; EDIL, T. B. Correlating index properties and hydraulic conductivity of geosynthetic clay liners. Journal of Geotechnical and Geoenvironmental Engineering, v. 131, n. 11, p. 1319-1329, 2005.

https://doi.org/10.1061/(ASCE)1090-0241(2005)131:11(1319)

LIN, L. C.; BENSON, C. H. Effect of Wet Dry Cycling on Swelling and Hydraulic Conductivity of GCLs. Journal of Geotechnical and Geoenvironmental Engineering, v. 126, n. 1, p. 40-49, 2000. https://doi.org/10.1061/(ASCE)1090-0241(2000)126:1(40)

MANASSERO, M.; BENSON, C. H.; BOUAZZA, A. Solid Waste Containment Systems. In: International Conference on Geological and Geotechnical Engineering (GeoEngineering2000), Melbourne, Australia. [Proceedings...]. v. 1, 2000, p. 520-642.

MAZZIERI, F.; PAQUALINI, E. Permeability of Damaged Geosynthetic Clay Liners. Geosynthetics International, v. 7, n. 2, p. 101-118, 2000. https://doi.org/10.1680/gein.7.0168

MEER, S. R.; BENSON, C. H. Hydraulic conductivity of geosynthetic clay liners exhumed from landfill final covers. Journal of Geotechnical and Geoenvironmental Engineering, v. 133, n. 5, p. 550-563, 2007. https://doi.org/10.1061/(asce)1090-0241(2007)133:5(550)

MELCHIOR, S.; SOKOLLEK, V.; BERGER, K.; VIELHABER, B.; STEINERT, B. Results from 18 years of in situ performance testing of landfill cover systems in Germany. Journal of Environmental Engineering, v. 136, n. 8, p. 815-823, 2010. https://doi.org/10.1061/ (ASCE)EE.1943-7870.0000200.

MENDES, M. J. A. Alguns Fatores que Influenciam o Desempenho de Geocompostos Bentoníticos sob Fluxo de Gases e Líquidos em Barreiras de Aterros Sanitários. 2010. Tese (Doutorado em Geotecnia) - Departamento de Engenharia Civil e Ambiental, Universidade de Brasília, DF, p. 180, 2010.

MIGUEL, G. D.; ENDLER, R. R.; FLOSS, M. F. Um Estudo da Mínima Razão de Cobrimento Adequada a Proteger Geocompostos Argilosos. Revista de Ciências Exatas Aplicadas e Tecnológicas da Universidade de Passo Fundo - CIATEC-UPF, v. 9, n. 2, p. 1-15, 2017. https://doi.org/10.5335/ciatec.v9i2.6442

MÜLLER, W.; JAKOB, I.; SEEGER, S.; TATZKY-GERTH, R. Long-term shear strength of geosynthetic clay liners. Geotextiles and Geomembranes, v. 26, n. 2, p. 130-144, 2008. https://doi.org/10.1016/j.geotexmem.2007.08.001 
PETROV, R. J.; ROWE, R. K.; QUIGLEY, R. M. Selected Factors Influencing GCL Hydraulic Conductivity. Journal of Geotechnical and Geoenviromental Engineering, v. 123, n. 8, p. 683695, 1997. https://doi.org/10.1061/(asce)1090-0241(1997)123:8(683)

PETROV, R.J.; ROWE, R.K. Geosynthetic clay liner (GCL) - chemical compatibility by hydraulic conductivity testing and factors impacting its performance. Canadian Geotechnical Journal, v. 34, n. 6, p. 863-885, 1997. https://doi.org/10.1139/t97-055.

ROUF, M. A.; BOUAZZA, A.; SINGH, R. M.; GATES, W. P.; ROWE, R. K. Gas flow unified measurement system for sequential measurement of gas diffusion and gas permeability of partially hydrated geosynthetic clay liners. Canadian Geotechnical Journal, v. 53, n. 6, p. 1000-1012, 2016. https://doi.org/10.1139/cgj-2015-0123

ROWE, R. K. Geosynthetics and the Minimization of Contaminant Migration Through Barrier Systems Beneath Solid Waste. In: Sixth International Conference on Geosynthetics, Atlanta. [Proceedings ...]. 1998, v. 1, p. 27-102.

ROWE, R. K. Geotechnical and Geoenviromental Engineering Handbook: Liner System. Springer US, Kluwer Academic Publishing, Norwell, USA, 2001. 1087 p.

https://doi.org/10.1007/978-1-4615-1729-0

ROWE, R. K. Long-term performance of contaminant barrier systems. Géotechnique, v. 55, n. 9, p. 631-678, 2005. https://doi.org/10.1680/geot.2005.55.9.631

ROWE, R. K. Performance of GCLS in liners for landfill and mining applications*. Environmental Geotechnics, v. 1, n. 1, p. 3-21, 2014. https://doi.org/10.1680/envgeo.13.00031

ROWE, R. K.; BRACHMAN, R. W. I.; HOSNEY, M. S.; TAKE, W. A.; ARNEPALLI, D. N. Insight into hydraulic conductivity testing of geosynthetic clay liners (GCLs) exhumed after 5 and 7 years in a cover. Canadian Geotechnical Journal, v. 54, n. 8, p. 1118-1138,

2017. https://doi.org/10.1139/cgi-2016-0473

ROWE, R. K.; BRACHMAN, R. W. I.; HOSNEY, M. S.; TAKE, W. A.; ARNEPALLI, D. N. Insight into hydraulic conductivity testing of geosynthetic clay liners (GCLs) exhumed after 5 and 7 years in a cover. Canadian Geotechnical Journal, v. 54, n. 8, p. 1118-1138, 2018.

https://doi.org/10.1139/cgj-2016-0473

SHACKELFORD, C.D.; BENSON, C.H.; KATSUMI, T.; EDIL, T. B.; LIN, L. Evaluating the hydraulic conductivity of GCLs permeated with non-standard liquids. Geotextiles and Geomembranes, v.18, n. 2-4, p. 133-161, 2000. https://doi.org/10.1016/S0266-1144(99)00024-2

SHUKLA, S. K.; YIN, J.-H. Fundamentals of Geosynthetic Engineering. Taylor \& Francis/Balkema, Leiden, The Netherlands, 2006. 410 p.

SIMON, F.-G.; MÜLLER, W. W. Standard and alternative landfill capping design in Germany. Environmental Science \& Policy, v. 7, n. 4, p. 277-290,

2004. https://doi.org/10.1016/i.envsci.2004.04.002

STARK, T. D.; ARELLANO, D.; EVAN, W. D.; WILSON, V. L.; GONDA, J. M. Unreinforced Geosynthetic Clay Liner Case History. Geosynthetics International, v. 5, n. 5, p. 521-544, 1998. https://doi.org/10.1680/gein.5.0135

VERTEMATTI, J. C. Manual brasileiro de geossintéticos. 1. ed., Blucher, São Paulo, Brasil, 2004. $413 \mathrm{p}$. 
VUKELIĆ, A.; SZAVITS-NOSSAN, A.; KVASNIčKA, P. The influence of bentonite extrusion on shear strength of GCL/geomembrane interface. Geotextiles and Geomembranes, v. 26, n. 1, p. 82-90, 2008. https://doi.org/10.1016/i.geotexmem.2007.04.001

ZANELLA, N. P. Comportamento do GCL permeado com Biodisel. 2019. Dissertação (Mestrado em Engenharia) - Programa de Pós-Graduação em Engenharia Civil e Ambiental, Universidade de Passo Fundo, RS, p. 88, 2019. 